\title{
Ground reference for space remote sensing
}

\section{Laboratory photometry of an asteroid model}

\author{
S. Kaasalainen ${ }^{1}$, M. Kaasalainen ${ }^{2}$, and J. Piironen ${ }^{3}$ \\ 1 Finnish Geodetic Institute, Department of remote sensing and photogrammetry, Geodeetinrinne 2, 02431 Masala, Finland \\ e-mail: Sanna .Kaasalainen@fgi.fi \\ 2 Department of Mathematics and Statistics, PO Box 68, 00014 University of Helsinki, Finland \\ e-mail: mjk@rni.helsinki.fi \\ 3 Finnish Geodetic Institute, Department of geodesy and geodynamics, Geodeetinrinne 2, 02431 Masala, Finland \\ e-mail: Jukka.Piironen@fgi.fi
}

Received 6 April 2004 / Accepted 25 May 2005

\begin{abstract}
We present accurate laboratory CCD photometry of an artificial asteroid sample. The standard inversion method for asteroid lightcurves was implemented to reproduce the measured lightcurves and to derive the global shape, spin state and pole direction to be compared with the known laboratory values. The results imply that the inversion analysis works universally for targets of arbitrary surface composition, i.e., the scattering law does not play a strong role in the analysis. We further investigated the scattering behaviour of the surface material by direct laboratory measurements, and found that the illumination phase angle dependence can be factorized for this material in the same way as for asteroids.
\end{abstract}

Key words. methods: laboratory - techniques: photometric - methods: numerical - scattering - minor planets, asteroids

\section{Introduction}

Laboratory measurements of asteroid models started with experiments carried out by Dunlap (1971). More detailed laboratory studies of asteroid models were accomplished more than ten years later by the System for Asteroid Models (SAM) at the Teramo Observatory, Italy (Barucci et al. 1982; D’Ambrosio et al. 1985), with a computerized system that allowed rotation and tilting of the model and a wide phase angle region. Laboratory models included meteorites, artificially produced fragments, cratered ellipsoidal figures and some dust covered models. SAM results showed the resemblance of asteroid lightcurves to the artificially produced lightcurves of collisional fragments (Barucci et al. 1983). Phase curve studies of the models were also carried out (e.g., Capaccioni et al. 1988). All the results indicated that asteroids have irregular shapes and that asteroids most probably have experienced a period of collisions. Although the SAM database was large, no inversion analysis was carried out, except for some attempts (e.g., Piironen et al. 1986). This was mostly due to the fact that no proper inversion procedures existed at the time. There were some attempts to use the SAM data to test rotational pole position determination methods (Barucci \& Fulchignoni 1982; Karttunen 1991). After this initial period of studies, no further laboratory experiments were made.
Our goals for this study are somewhat different from those of the earlier studies. In situ and radar imaging data are now available (Ostro et al. 2002; Sullivan et al. 2002; Kaasalainen et al. 2002a), various kinds of shapes and surface structures can readily be studied with computer simulations, and exact lightcurve inversion can routinely be performed (Kaasalainen et al. 2001, 2002b). Thus we now know what kinds of targets cause the observed lightcurves, and we know what kinds of lightcurves are produced by arbitrary numerical models. What is still needed for accurate and reliable space remote sensing is a range of well-defined and controlled ground truths. This can be achieved either with asteroid orbiter missions or by laboratory studies. Orbiters are scarce: so far, detailed photometric studies are only available from NEAR at Eros, while even better results are expected from Hayabusa at Itokawa. Laboratories constitute an artificial environment, but with them we can create a complete link from surface material to observations.

The main goals of this paper are to show that:

1. Efficient and accurate laboratory measurements $(S / N \sim$ 400 for relative photometry) are easy to implement for this kind of ground reference study.

2. The surface material is not crucial in the analysis: inversion techniques produce reliable results even for a clay/sand surface.

3. Scattering characteristics can be separately and directly measured for the surface material. 


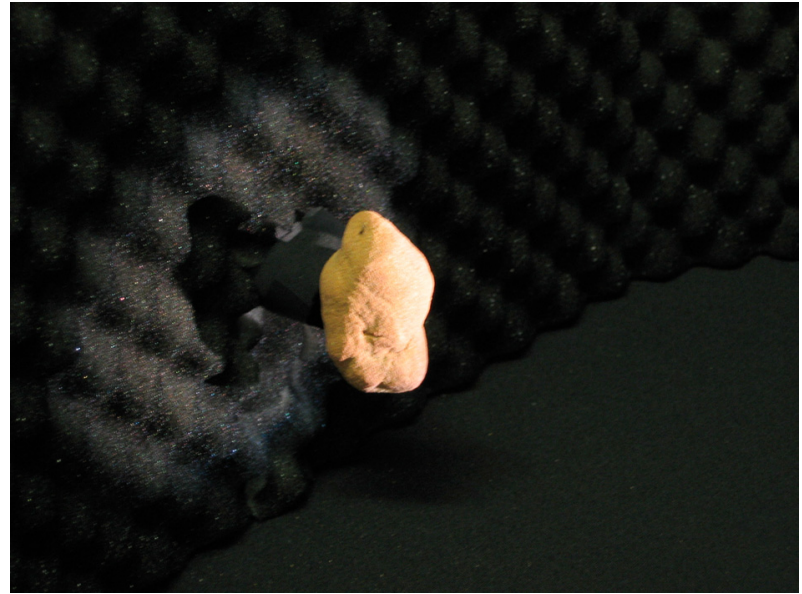

Fig. 1. The sample mounted in its rotator against the background. The illumination (lamp) spot is also visible.

\section{The measurements}

To produce laboratory lightcurves for a simulated asteroid, we constructed a prototype instrument for lightcurve photometry. The setup consisted of a stabilized $50 \mathrm{~W}$ quartz-tungstenhalogen $(\mathrm{QTH})$ broadband source (Oriel), with $2 \mathrm{~mm}$ aperture (to simulate a point source) and a CCD camera with $50 \mathrm{~mm}$ objective $(\mathrm{F} / 16)$, mounted on a tripod so that its position could be varied. The sample was mounted on a precision rotator which permitted $360^{\circ}$ rotation. The light source and camera positions could be changed to vary the illumination and observation geometry, whereas the asteroid position and rotation axis were fixed. The sample was placed in the centre of an about $12 \mathrm{~cm}$ wide lamp spot (Fig. 1) to allow for uniform illumination over the sample and background (matt black painted foam). Because the camera position was held fixed during the measurement of each lightcurve, the only varying parameter was the rotational position and hence the brightness of the object itself. The target brightness was summed over the entire image and divided by the number of pixels in the frame. Each point in a lightcurve is an average of 3 camera exposures of the illuminated sample, and the measurement error in terms of their standard deviations was less than $0.3 \%$.

The sample (Fig. 1) was made of Das modelling clay and coated with Sahara sand taken from Assuan, Egypt (see Fig. 2 for the grain size estimation). The average dimensions of the sample were about $6.5 \times 2.5 \mathrm{~cm}$. The approximate roughness scale (if we assume, e.g., a $0.1 \mathrm{~mm}$ average grain size) is thus about 1/500. For comparison, this reduced to, e.g., a $10 \mathrm{~km}$ sized object would mean about a $20 \mathrm{~m}$ roughness scale for the surface features (though we do not intend to simulate a "real" asteroid surface). The average size/distance scale of the object caused the incident light direction to spread over an angle of about $1^{\circ}-2^{\circ}$, which had no photometric significance.

To investigate the scattering properties of the surface material of the sample asteroid directly and quantitatively, we measured the bidirectional reflectance function (BRDF) for a sand-covered disc (of about $50 \mathrm{~cm}$ in diameter) prepared in the same way as the sample, using a spectrogoniometer with ASD FieldSpec Pro spectrometer and a $1000 \mathrm{~W}$ Oriel research

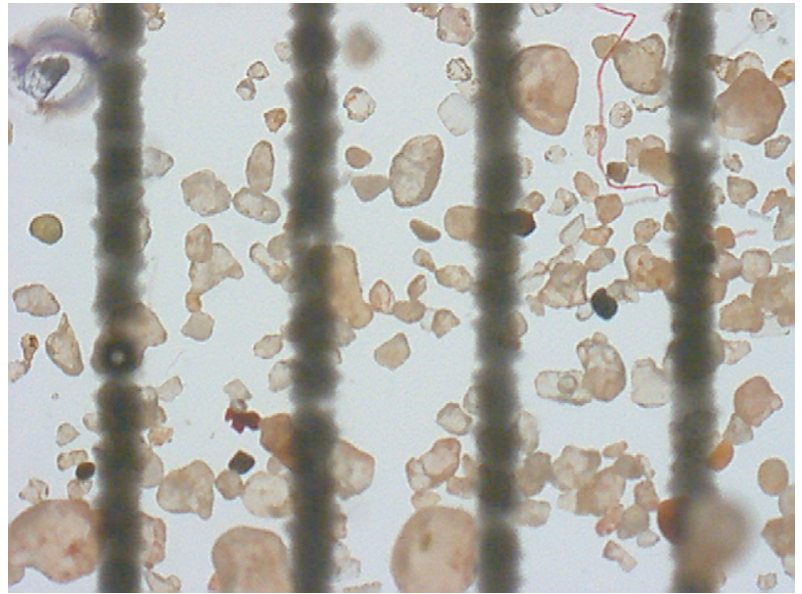

Fig. 2. Microscopic image of sand which was used to cover the sample. The scale is in $\mathrm{mm}$.

QTH lamp (Peltoniemi et al. 2005). The reflectances at phase angles $<10^{\circ}$ were measured with a small-angle goniometer constructed particularly for backscattering measurements (see Kaasalainen et al. 2005). All scattering measurements are relative to a Spectralon (Labsphere Inc.) reference plate, which is roughly similar to a Lambert surface in scattering properties. In these terms the albedo of the sample material is roughly $0.5-0.6$ (Fig. 7b).

\section{Results}

\subsection{Shape and spin state}

Numerical simulations of the lightcurves of various objects and surface or scattering types and the related inversion results are now a well-studied subject (see, e.g., Durech \& Kaasalainen 2003; Kaasalainen et al. 2004, and the references therein). Investigating a large set of objects in a laboratory is thus not our objective. We want to compare the results from an arbitrary and a priori unknown scattering surface with numerical simulations and thus to perform the basic check on whether the implications from the latter are indeed generally valid, particularly with a small number of data points. As checked in Kaasalainen et al. (2001), the inversion method is consistent with the ground truth from NEAR and Galileo missions, but our aim here is to use non-asteroidal material and the laboratory environment to check the universality of the method and to facilitate a close inspection of the surface material.

We chose seven different observing geometries for the target; their phase angles $\alpha$ and viewing/illumination aspects $\theta / \theta_{0}$ measured from the pole are shown in Fig. 3, where the observed intensities are given as asterisks and the inversion model lightcurves as dashed lines. The photometry was relative (uncalibrated) and unfiltered. The observation "epochs" were defined by the readings of the rotation angle scale fixed to the axis of the sample asteroid. The scale of these epochs is arbitrary; we chose one degree to represent one minute, corresponding to a period of six hours. The intervals between the lightcurves are also irrelevant for this study as we did not need to mimic any celestial motion: the problem is a mathematical one related to 

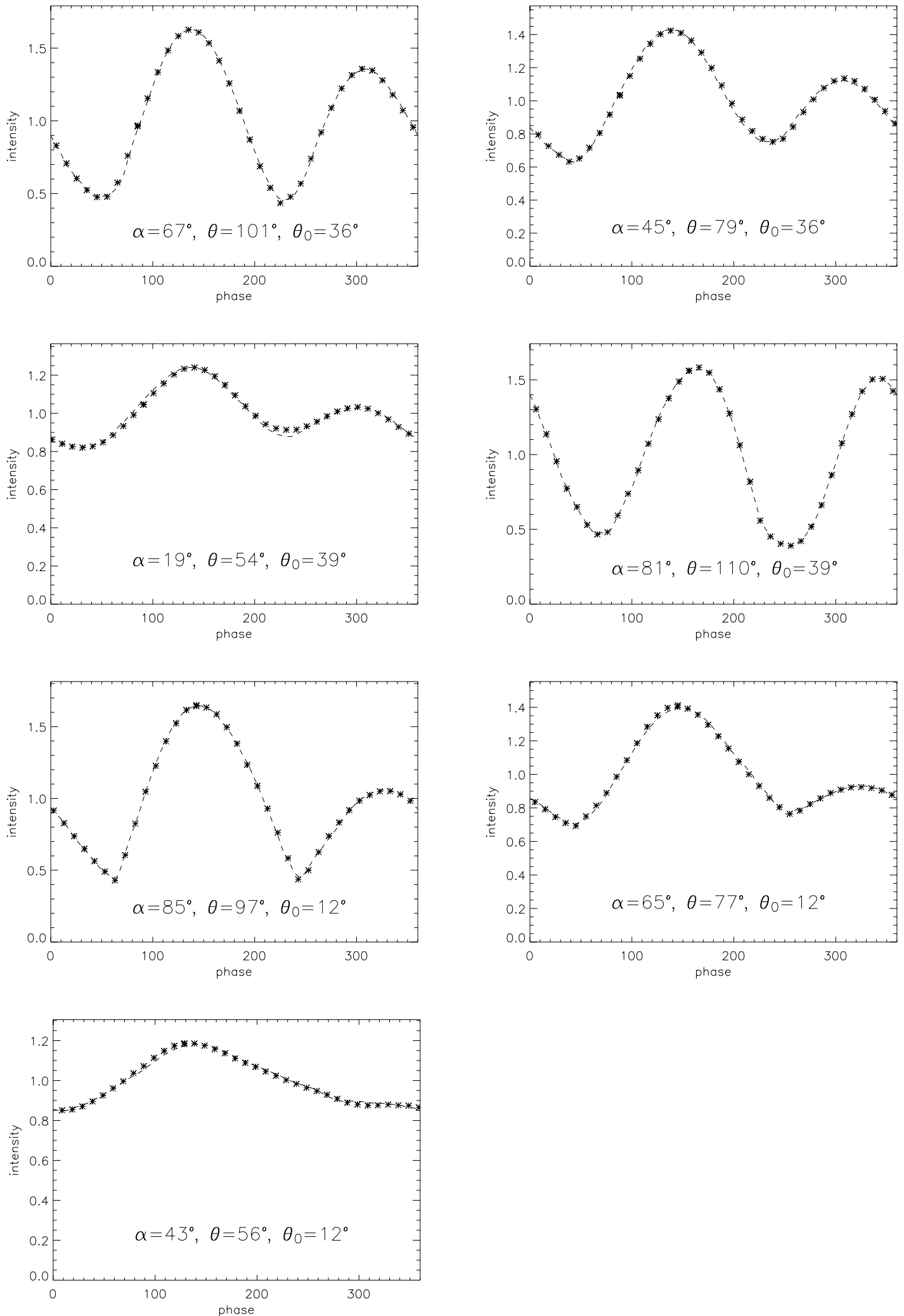

Fig. 3. Measured and modelled lightcurves. Measured points are shown as asterisks, and the model reproduction as a dashed line. Intensity is in relative units.

varying observing geometries around a uniformly rotating object. Furthermore, the phase angles (up to $85^{\circ}$, see Fig. 3) were not realistic for main-belt asteroids, as our objective was rather to demonstrate the case with a limited, but geometrically wideranging data set (which is why we also implemented a nearly ecliptic pole position). Phase angles this large can be observed 
for near-Earth asteroids (Kaasalainen et al. 2004). An interesting future issue would be to investigate, e.g., a near poleupright case at phase angles typical of main-belt asteroids.

The inversion analysis was carried out with the standard technique of Kaasalainen et al. (2001, 2002a). A remarkable fact is that the inversion was successfully performed with the same scattering laws as generally used in space remote sensing, i.e., a combination of the Lommel-Seeliger and Lambert laws or the Hapke scattering model (see Kaasalainen et al. 2004, and references therein), even though these were not originally designed for this kind of surface. As shown in Fig. 3, the inversion model reproduces the observed lightcurves extremely well (down to $1 \% \mathrm{rms}$ deviation). Images of the resulting global convex shape are shown (in Fig. 4) together with photographs of the target (in Fig. 5). The basic characteristics of the shape are surprisingly well revealed from only 252 data points. The ratio of the largest length/width extents is 1.95 for the model and 2.15 for the target; the $10 \%$ "shortening" of the model is caused by the obviously inaccurate scattering model. Similarly, the model is slightly flattened in the vertical direction. As could be expected, nonconvex inversion did not converge to as good a chi-square and did not produce unambiguous details, consistent with Kaasalainen et al. (2001) and Durech \& Kaasalainen (2003).

The spin state was accurately determined within a few degrees of the correct value: the (most often used) relative Lambert/Lommel-Seeliger ratio 0.1 gave the pole direction as $\lambda=34 \pm 5^{\circ}, \beta=-3 \pm 3^{\circ}$, while the ratio 0.3 gave $\lambda=$ $28 \pm 5^{\circ}, \beta=-3 \pm 3^{\circ}$ with as good a fit. The measured pole position was $\lambda=26 \pm 4^{\circ}, \beta=-1 \pm 3^{\circ}$, so the higher Lambert ratio may reflect the fact that the albedo of the laboratory target is much higher than that of the dark solar system bodies approaching the Lommel-Seeliger approximation. The Hapke scattering model did not allow good convergence if the parameters were left free, but freezing the parameters to those of average S-type asteroids and using $\bar{\theta}=40^{\circ}$ for the surface roughness parameter led to as good a fit as above, with the pole solution $\lambda=29 \pm 5^{\circ}, \beta=-3 \pm 3^{\circ}$. The rotation period was uniquely determined to be 6 hours within the time-line accuracy rule of Kaasalainen et al. (2001) (here the chosen length of the time line and hence the accuracy of the period is arbitrary).

To explore the minimal information requirement, we also carried out the inversion using only three lightcurves, the ones at phase angles $19^{\circ}, 43^{\circ}$ and $81^{\circ}$, while reducing the density of lightcurve points to half. Using only these 54 data points from widely varying observing geometries still yields a unique pole direction of $\lambda=30 \pm 8^{\circ}, \beta=-2 \pm 6^{\circ}$ and an approximate global shape that clearly shows the asymmetric character (pole view in Fig. 6). This clearly demonstrates that the information content of photometry is indeed very high as far as the basic characteristics of the target are concerned.

\subsection{Scattering properties}

The scattered intensity of a surface patch of an object is typically expressed in the form $S\left(\mu, \mu_{0}, \alpha\right)$, where $\mu$ and $\mu_{0}$ are the normal cosines of observation and illumination directions,

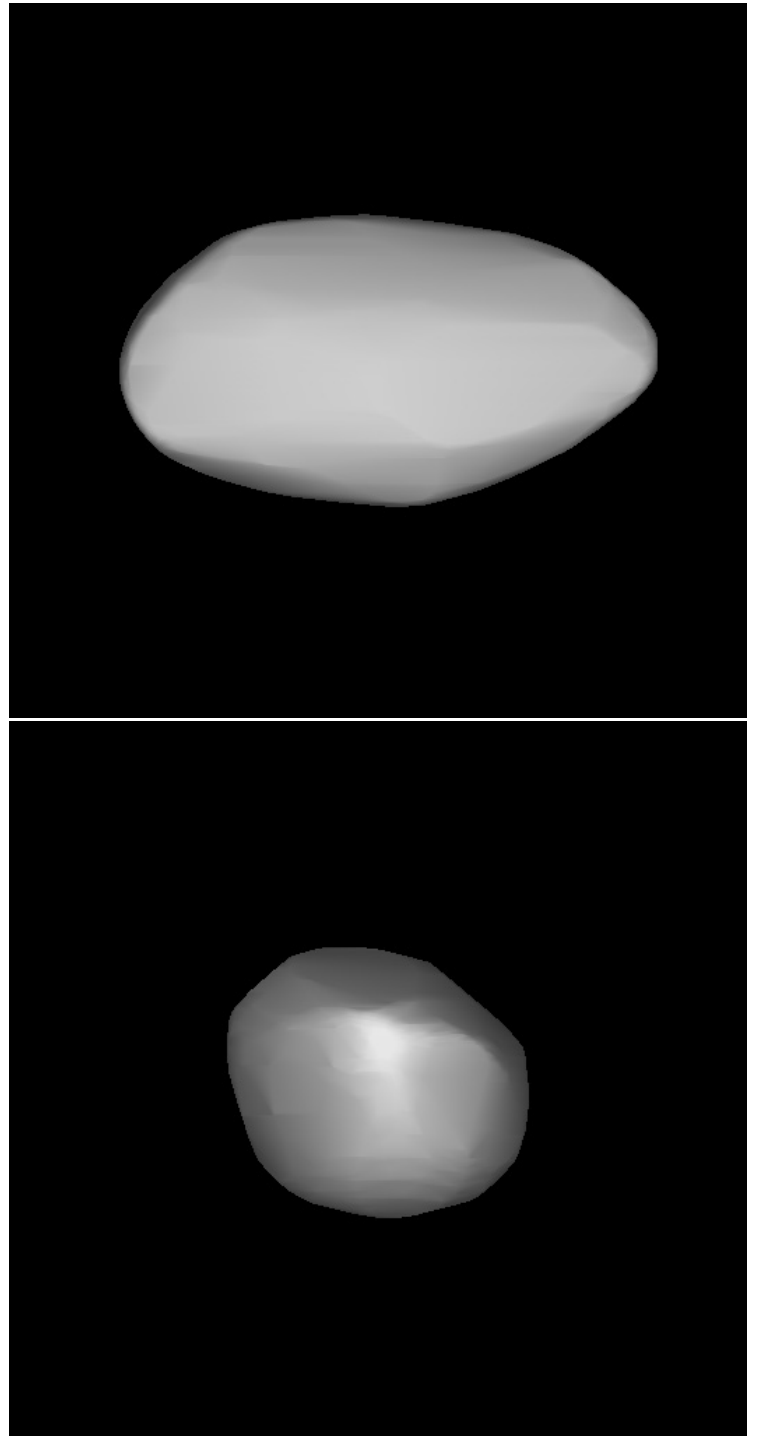

Fig. 4. Views of the shape model directly from above and from the equatorial level. The shape was obtained with only 252 data points.

respectively, and $\alpha$ is the angle between these directions (i.e., the phase angle). A standard BRDF measurement for the target's surface material was made at a fixed $\mu_{0}$ (due to the limited opportunity to vary the $\mu_{0}$ in other than a narrow range of incidence angles in the goniometer used for the measurement). Figure $7 \mathrm{a}$ shows the measured reflectances (intensities) plotted as a function of phase angle at different values of $\mu$, corresponding to emergence angles $40^{\circ}$ (pluses), $50^{\circ}$ (asterisks) and $60^{\circ}$ (diamonds). The incidence angle was $65^{\circ}$, which sets the limits for the phase angle ranges for each fixed $\mu$.

Figure $7 \mathrm{a}$ suggests that the phase behaviour is essentially the same at each $\mu$, so the scattering law $S$ can be factorized as $f(\alpha) S\left(\mu, \mu_{0}\right)$ as in, e.g., Kaasalainen et al. (2001). The $S\left(\mu, \mu_{0}\right)$-part should obviously decrease as $\mu$ decreases, but apparently the decrease is here neither as uniform nor as fast as in the Lommel-Seeliger and Lambert combination. The $S\left(\mu, \mu_{0}\right)$ factor appears to be essentially at the same level at all values of $\mu$, which mainly results from the way the BRDF measurements are made in standard goniometry for plane surfaces (cf. 


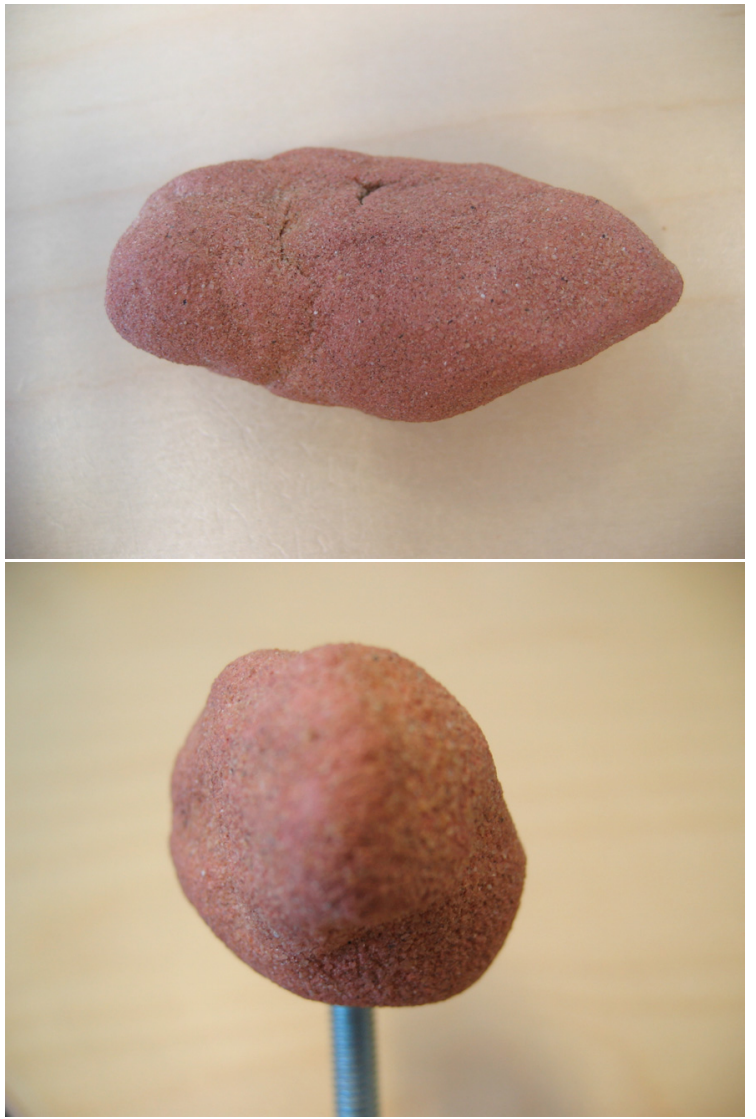

Fig. 5. Views of the target from the same viewpoints as the model images (Fig. 4).

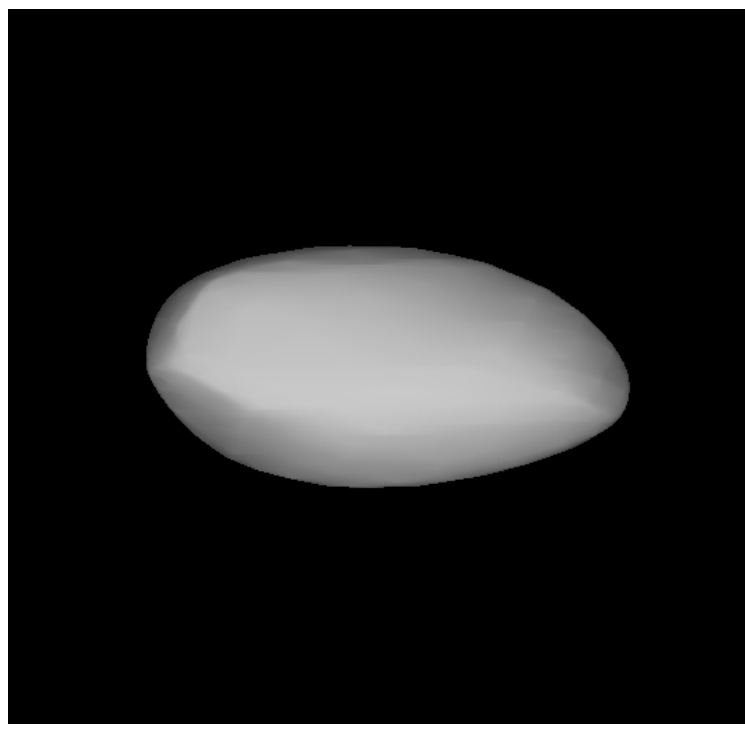

Fig. 6. Even 54 well-distributed points give a good basic shape model.

Peltoniemi et al. 2005), where the elongation of the detector field of view compensates for the differences caused by projection (i.e. the $\mu$ variation) at large zenith angles. A more complete characterization of the scattering law would require an advanced laboratory experiment with varying $\mu_{0}$ and comparable $\mu$-levels. The current result, however, serves as an example of scattering behaviour that can be factorized. To illustrate the
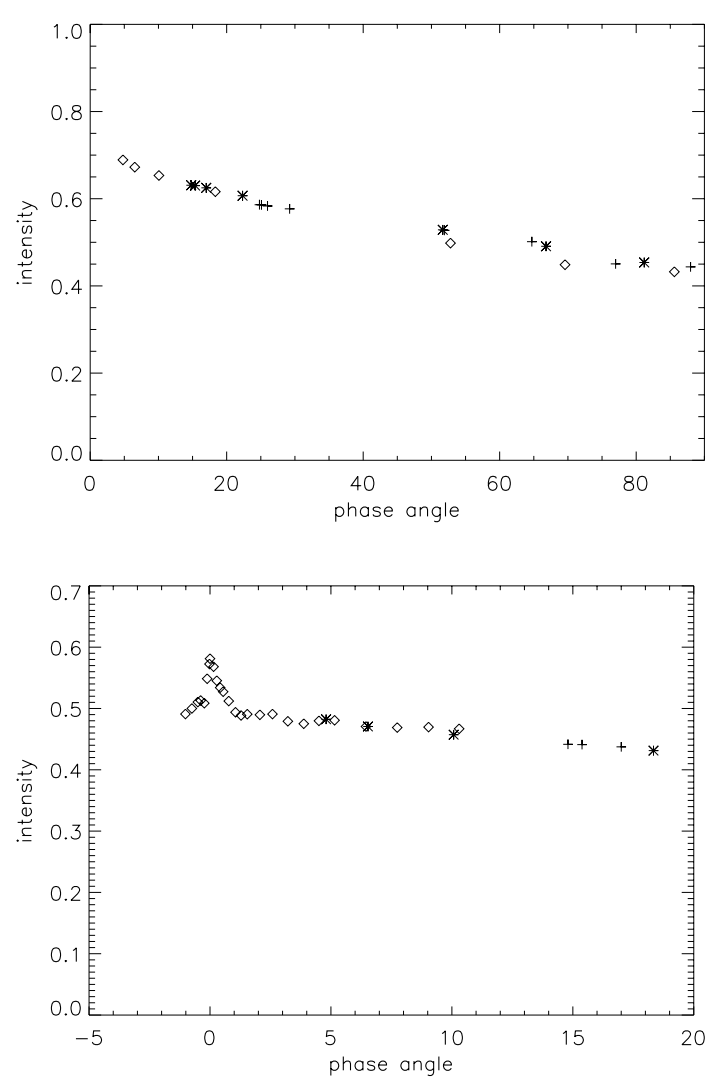

Fig. 7. a) The measured BRDFs at different values of $\mu$, at $633 \mathrm{~nm}$. b) Phase curves at $633 \mathrm{~nm}$ combined (by normalizing at $6.5^{\circ}$ ) from BRDF measurements (asterisk; the plot is made at $\mu=60^{\circ}$ ) and smallangle laser measurement (diamond).

phase behaviour of the surface material at phase angles down to $0^{\circ}$, we combined the phase curve obtained from the BRDF measurements with that measured with a laser-based smallangle goniometer (see Kaasalainen et al. 2005). The combined plot, presented in Fig. 7b, shows an opposition effect similar to those measured in the laboratory for regolith analog materials (e.g., Kaasalainen 2003). (The negative phase angles in the plot denote observations in the direction opposite to the positive one.)

As mentioned above, the striking fact is that, despite their inadequacy here, the asteroidal scattering laws could be succesfully used to obtain the spin state and characteristic shape of the object. This clearly underlines the two central aspects of lightcurve inversion:

1. The inversion procedure is very stable against insufficient knowledge of the scattering law. From its point of view, scattering behaviour is roughly similar for a variety of surface materials, ranging from sand/clay to asteroid regolith.

2. However, it is in practice impossible to obtain any nonconvex shape features (unless they are very large and the phase angle is very high) or other details since this would require very accurate knowledge of the scattering properties everywhere on the surface.

Thus, photometric data are remarkably rich in information, but there is also a definite limit to this information. 


\section{Conclusions}

The main conclusions of this study are:

1. We have shown that a laboratory approach together with the inversion analysis presented is a robust method of obtaining ground truth for remote sensing of asteroids.

2. Photometry does not contain information on local features of the shape: a global convex shape typically fits observations perfectly, even at large phase angles when using an imperfect scattering law (cf. Durech \& Kaasalainen 2003).

3. The scattering law does not play a key role in inversion. The spin state and global shape determine the lightcurves almost completely, and the only properly extractable component of the scattering law is the phase function $f(\alpha)$ with calibrated photometry.

4. Even a very small data set covering a wide range of observing geometries contains a remarkably large amount of information on the shape and spin state of the target.

This study is an example of how even an artificial laboratory case study can provide a feasible means for obtaining ground truth for space remote sensing methods. As most of the target characteristics are universal, it is possible to simulate them in the laboratory, especially when there is limited opportunity for their direct and detailed investigation in space. In future studies, it would be very interesting to obtain experimentally a more detailed scattering law $S\left(\mu, \mu_{0}, \alpha\right)$ and use this in inversion.

Acknowledgements. Part of this work was supported by the Academy of Finland. Thanks to Juha Suomalainen at the FGI for help with the scattering measurements and Jouni Peltoniemi at the FGI for the Sahara sand.

\section{References}

Barucci, M., \& Fulchignoni, M. 1982, Moon and the Planets, 27, 47

Barucci, M., Casacchia, R., Fulchignoni, M., et al. 1982, Moon and the Planets, 27, 387

Barucci, M., Capaccioni, F., Cerroni, P., Flamini, E., \& Fulchignoni, M. 1983, in Asteroids, Comets, Meteors, Uppsala University, 95

Capaccioni, F., Barucci, M., Cerroni, P., \& Fulchignoni, M. 1988, in Lunar and Planetary Science Conference abstracts, 164

D’Ambrosio, V., Burchi, R., Paolantonio, A. D., \& Giuliani, C. 1985, A\&A, 144, 427

Dunlap, J. 1971, in Physical Studies of Minor Planets, ed. T. Gehrels, NASA SP-267, 147

Durech, J., \& Kaasalainen, M. 2003, A\&A, 404, 709

Kaasalainen, M., Torppa, J., \& Muinonen, K. 2001, Icarus, 153, 37

Kaasalainen, M., Mottola, S., \& Fulchignoni, M. 2002a, in Asteroids III, ed. W. Bottke, R. Binzel, P. Paolicchi, \& A. Cellino (Tucson: The University of Arizona Press), 139

Kaasalainen, M., Torppa, J., \& Piironen, J. 2002b, Icarus, 159, 369

Kaasalainen, M., Pravec, P., Krugly, Y., et al. 2004, Icarus, 167, 178

Kaasalainen, S. 2003, A\&A, 409, 765

Kaasalainen, S., Peltoniemi, J., Näränen, J., et al. 2005, Appl. Opt., 44, 1485

Karttunen, H. 1991, A\&A, 244, 553

Ostro, S. J., Hudson, R. S., Benner, L. A. M., et al. 2002, in Asteroids III, ed. W. Bottke, R. Binzel, P. Paolicchi, \& A. Cellino (Tucson: The University of Arizona Press), 151

Peltoniemi, J., Kaasalainen, S., Näränen, J., et al. 2005, Remote Sensing of Environment, 94, 343

Piironen, J., Karttunen, H., Lumme, K., Barucci, M., \& Fulchignoni, M. 1986, BAAS, 18,801

Sullivan, R. J., Thomas, P. C., Murchie, S. L., \& Robinson, M. S. 2002, in Asteroids III, ed. W. Bottke, R. Binzel, P. Paolicchi, \& A. Cellino (Tucson: The University of Arizona Press), 331 